\title{
Measuring Outer Disk Warps with Optical Spectroscopy
}

\author{
Daniel Christlein $^{1}$ and Joss Bland-Hawthorn ${ }^{2}$ \\ ${ }^{1}$ Max-Planck-Institut für Astrophysik, \\ Karl-Schwarzschild-Str. 1, 85748 Garching, Germany \\ email: dchristl@mpa-garching.mpg.de \\ ${ }^{2}$ Institute of Astronomy, School of Physics \\ University of Sydney, NSW 2006, Australia \\ email: jbh@physics.usyd.edu.au
}

\begin{abstract}
Warps in the outer gaseous disks of galaxies are a ubiquitous phenomenon, but it is still unclear what generates them. One theory is that warps are generated internally through spontaneous bending instabilities. Other theories suggest that they result from the interaction of the outer disk with accreting extragalactic material. In this case, we expect to find cases where the circular velocity of the warp gas is poorly correlated with the rotational velocity of the galaxy disk at the same radius. Optical spectroscopy presents itself as an interesting alternative to 21-cm observations for testing this prediction, because (i) separating the kinematics of the warp from those of the disk requires a spatial resolution that is higher than what is achieved at $21 \mathrm{~cm}$ at low HI column density; (ii) optical spectroscopy also provides important information on star formation rates, gas excitation, and chemical abundances, which provide clues to the origin of the gas in warps. We present here preliminary results of a study of the kinematics of gas in the outer-disk warps of seven edge-on galaxies, using multi-hour VLT/FORS2 spectroscopy.
\end{abstract}

Keywords. galaxies: evolution, galaxies: formation, galaxies: kinematics and dynamics, galaxies: structure

\section{Introduction}

Warps in the outer disks of galaxies are a ubiquitous phenomenon. They are seen both in the distribution of stars (Sanchez-Saavedra et al. 1990; Cox et al. 1996) and neutral hydrogen (e.g., Sancisi 1976; Bosma 1981), and surveys estimate that possibly $50 \%$ or more of all galaxies show evidence for warps beyond the isophotal radius $\mathrm{R}_{25}$ (Briggs 1990), which typically contains $\sim 90 \%$ of the total light. Usually, these manifest themselves in the form of the outer disk bending away from the plane defined by the inner disk, defining either the shape of a bowl, or, more commonly, an integral sign as seen edge-on. This suggests that specific angular momentum of material in the warps is not aligned with that of the inner disk.

Numerous suggestions have been made over the years for the responsible mechanisms. Among the earliest such proposals were internal bending modes in the disk (LyndenBell 1965), but such modes were soon recognized to be persistent only in a disk with an unrealistically sharp mass truncation (Hunter \& Toomre 1969). Revaz \& Pfenniger (2004) have revived this discussion by identifiying short-lived bending instabilities as a possible cause. Other proposed mechanisms focus on the interaction of the baryonic disk with its environment: A bending of the outer baryonic disk may be introduced by a misalignment between the angular momentum of the inner baryonic disk and the hypothetical nonspherical, dark matter halo that it is embedded in (Toomre 1983; Dekel \& Shlosman 1983; Kuijken 1991; Sparke \& Casertano 1998), creating a gravitational torque on the 
disk. However, it has been argued that the inner halo would realign with the baronic disk over time, and the warp would dissipate (Nelson \& Tremaine 1999; Binney, Jiang \& Dutta 1998; Dubinski \& Kuijken 1995; New et al. 1998). Ostriker \& Binney (1989), Jiang \& Binney (1999), Shen \& Sellwood (2006) consider the impact of ongoing accretion onto an outer dark matter halo and argue that, since the angular momentum of infalling material will in general not be aligned with the present galaxy disk, this will create an ongoing torque on the outer galaxy disk. Binney (1992) has furthermore suggested that, if infalling material loses angular momentum to the halo, it might penetrate as far as the outer edge of the baryonic disk itself.

It is this hypothesis that we wish to test with the present work. How galaxies acquire gas is one of the key questions in our understanding of how they evolve, and determining whether warps are indeed signatures of such processes therefore is of great importance.

How can the direct accretion hypothesis be tested? The specific angular momentum vector of accreting material will, in general, neither be aligned exactly with that of the inner disk, nor have the same size. If such infalling material is indeed in direct contact and exchanging angular momentum with gas in the outermost baryonic disk, then it will introduce kinematic anomalies, i.e., a deviation from disk-like rotation, such as a lag or excess in the circular velocity. Measuring the circular velocity of gas in the warps therefore becomes an important observational discriminator.

Our project has measured line-of-sight velocities of gas in the outer disks of seven galaxies, the majority of which display clear signs of optical warps. Our measurements were obtained via optical spectroscopy of the $\mathrm{H} \alpha$ line. Although the tradititional way of observing gas in the outer disk is via the 21-cm line of neutral hydrogen, optical spectroscopy has proven a surprisingly successful alternative for studying the outer disk (Bland-Hawthorn, Freeman \& Quinn 1997; Christlein \& Zaritsky 2008), for a number of reasons: 1) Most importantly, the spatial resolution - an order of magnitude better than even the highest-quality interferometric HI maps - allows us to clearly separate gas in the warps from gas in the plane of the galaxy. This, in turn, allows us to access galaxies with smaller angular diameters at larger redshifts, greatly increasing the number of suitable targets. 2) Sporadic local star formation or illumination of gas in the warps by escaping UV flux from the inner star-forming disk guarantee low-level $\mathrm{H} \alpha$ flux from the outer disk far beyond what is usually perceived as the star formation threshold. It has been demonstrated (Bland-Hawthorn, Freeman \& Quinn 1997; Christlein \& Zaritsky 2008) that multi-hour optical spectroscopy of such low-level emission can, in some cases, probe outer galaxy disks to similar extents as 21-cm. 3) Optical spectroscopy yields a plethora of ancillary data, particularly stellar continuum and metal lines. In determining the origin of gas in the outer disks and warps, metallicity indicators may provide valuable additional insight (although an analysis exceeds the scope of these proceedings). Our aim is to a) measure the rotational velocity of gas in the warps and b) determine whether there are kinematic anomalies in this rotational velocity, i.e., sudden breaks or upturns that are not consistent with an extrapolation of the rotation curve from the inner disk, which may be associated with the onset of the warp.

\section{Observations}

Our sample consists of seven galaxies, of which six were taken from the catalog of optically warped galaxies by Sanchez-Saavedra et al. (2004); one object was targetted blindly without prior knowledge of a warp. All are nearly edge-on, with redshifts in the range of several thousand $\mathrm{km} / \mathrm{s}$, and their angular size is well-matched to the field of view. 


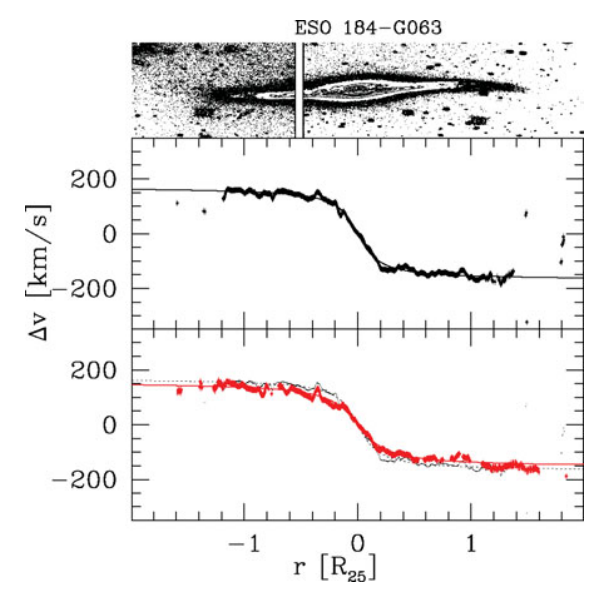

Figure 1. ESO 184-G063. For explanation, see text. $\mathrm{H} \alpha$ is more extended in the offset spectrum than along the major axis, but no kinematic disruption is associated with the warp.

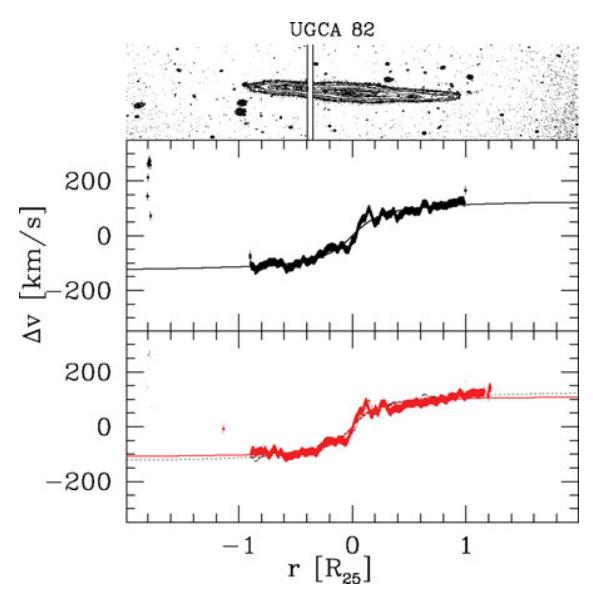

Figure 2. UGCA 23. For explanation, see text. $\mathrm{H} \alpha$ is more extended in the offset spectrum than along the major axis, but no kinematic disruption is associated with the warp.

Our observations were carried out over three nights in September 2007, using FORS2 on the VLT-UT1. The typical observing strategy was to observe for a total of one hour with a long slit of $0.5^{\prime \prime}$ width aligned along the major axis (to obtain a reference for the rotational velocity and angular extent gas in the plane of the galaxy), then for two hours with a position angle offset from the major axis PA by a few degrees. The purpose of the latter observation was to assure that $\mathrm{H} \alpha$ emission emanating from the warps would be observed, rather than from the plane of the galaxy. Sense and size of this offset were determined on a case-by-case basis after inspecting plates from the Digitzed Sky Survey as well as our own acquisition images, and typically chosen so that the slit would pass within or just beyond the outermost contours of stellar continuum light at the end of the warp.

\section{Results}

- ESO 184-G063 (Fig. 1) is a small ( $M=-18.5)$ Sb-type galaxy at cz $=3207 \mathrm{~km} / \mathrm{s}$, and has a strong integral-sign warp, which is distinctly stronger on one side than the other.

This figure, as well as all subsequent ones, shows the rotation curve along the major axis (top panel) and in the offset position (bottom panel; major axis rotation curve plotted with small dots/dashed lines for reference). The radial extent is $2 R_{25}$ on both sides of the nucleus; the acquisition image is plotted to the same scale.

Along the major axis, our rotation curve extends to $1.4 \mathrm{R}_{25}$, which in itself is remarkable, given that normal $\mathrm{H} \alpha$ rotation curves with conventional exposure times and smaller instruments typically reach $0.7 R_{25}$, and rarely as far as $R_{25}$. Our off-axis spectrum extends out to $1.6 \mathrm{R}_{25}$, clearly intersecting the tip of the optical warp. Both the major axis and the offset rotation curves are consistent, and there is no sign of a sudden break in the rotational velocity coincident with the onset of the warp.

- UGCA 23 (Fig. 2) is an $M=-19$ Sd-type galaxy at cz=3864 km/s. Acquisition images show lumpy emission and slightly bent isophotes on one side. The major axis rotation curve extends to $\sim R_{25}$ in this case. The offset spectrum samples the warp feature 


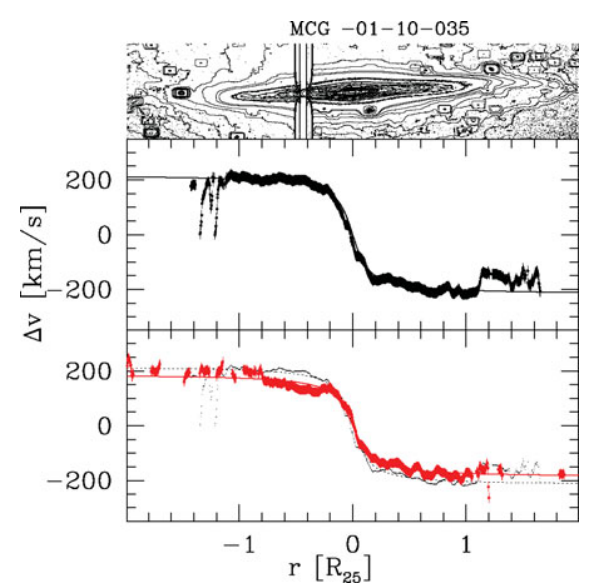

Figure 3. MCG -01-10-035. H $\alpha$ is more extended in the offset spectrum than along the major axis. A break in the rotation curve is found along the major axis, coinciding with the onset of a tidal-tail-like warp feature.

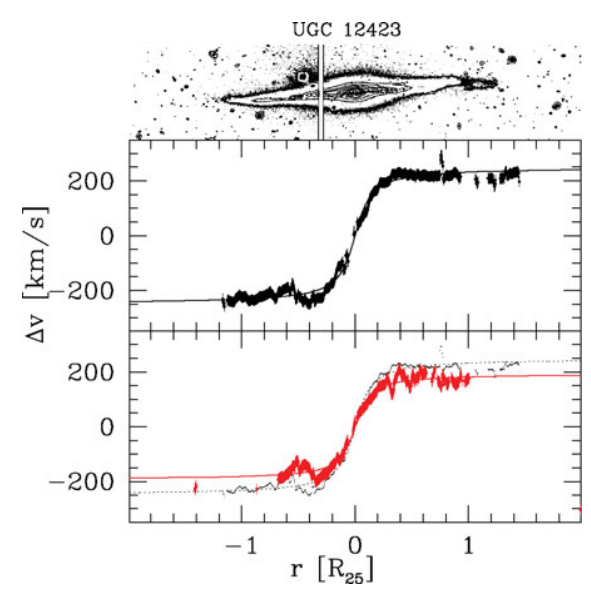

Figure 4. UGC 12423. See text for explanation.

and shows emission as far as $1.2 \mathrm{R}_{25}$. The two rotation curves are indistinguishable; the kinematics of the extraplanar feature are disk-like.

- MCG -01-10-035 (Fig. 3) is a small ( $M=-18.3)$ Sc-type galaxy at cz $=3788$ $\mathrm{km} / \mathrm{s}$. Our acquisition images reveal a very extended tail of low surface brightness extending as far as $2 R_{25}$ and then curving back. Its appearance is very suggestive of a tidal feature. While the other side of the galaxy does not exhibit an equivalently striking feature, the outer isophotes appear bent on both sides.

The major axis spectrum samples far into this low-surface brightness tail and extends to $1.6 \mathrm{R}_{25}$ on that side, and $1.4 R_{25}$ on the other. Remarkably, at the onset of this feature, around $\mathrm{R}_{25}$, we see a sudden break in the rotation curve along the major axis in the sense of a jump to lower rotational velocities by $\sim 60-70 \mathrm{~km} / \mathrm{s}$. On the other side, no such strong break is discernible, but there is $\mathrm{H} \alpha$ emission at velocities distinctly below the extrapolated rotation velocity, going as far down as the systemic velocity of the galaxy itself.

Our offset spectrum intercepts the tail at its largest extent. While we do not see a continuous $\mathrm{H} \alpha$ signal beyond $\mathrm{R}_{25}$, there are individual $\mathrm{H} \alpha$-bright regions extending as far as $2 R_{25}$ on both sides of the galaxy. The rotation curve fitted to this spectrum shows no breaks in the velocity; however, at large radii, its line-of-sight velocity is consistent with the lower-velocity gas in the tidal feature.

- UGC 12423 (Fig. 4) is an Sc-type galaxy at cz $=4839 \mathrm{~km} / \mathrm{s}$ with an absolute magnitude $M=-19.4$. It shows a strong and asymmetrical optical warp. The majoraxis rotation curve extends to $1.4 \mathrm{R}_{25}$ on the side with the stronger warp, which is even farther than the extent of the warped disk. At the outermost point, the rotational velocity is still consistent with the extrapolated rotation curve. A possible dip in the rotation velocity at the position of the warp feature does not appear significant.

The offset spectrum is, in this case, much less extended; it reaches $R_{25}$ on the side of the stronger warp, and only $0.7 \mathrm{R}_{25}$ on the other side, with the exception of one small $\mathrm{H} \alpha$-emitting region at $1.4 \mathrm{R}_{25}$. There are no signs for any kinematic anomalies in this rotation curve. 


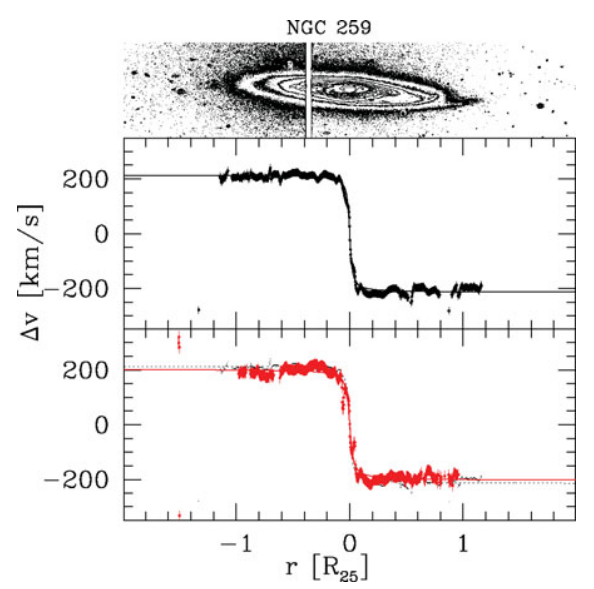

Figure 5. NGC 259. See text for explanation.

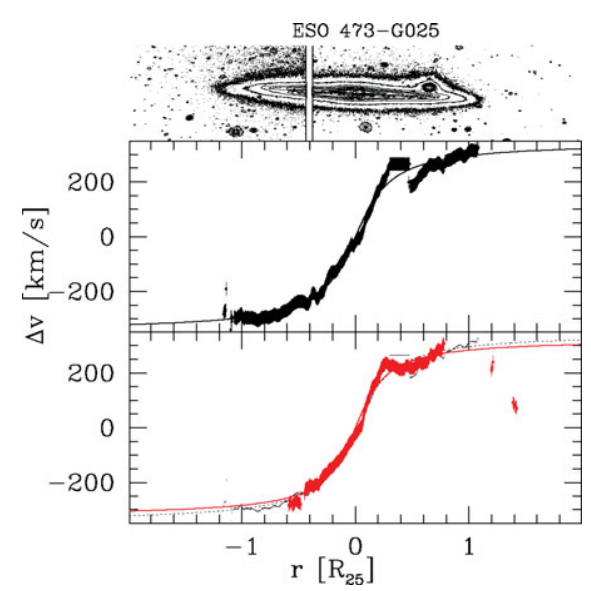

Figure 6. ESO 473-G025. text for explanation.

- NGC 259 (Fig. 5) is among the brighter galaxies in this sample $(M=-20)$, an Sbc-type at $c z=4045 \mathrm{~km} / \mathrm{s}$. It is also the least inclined among our targets. The acquisition images show that the supposed warp feature is in fact more suggestive of a continuation of a spiral arm. The major axis spectrum samples its kinematics out to $1.2 \mathrm{R}_{25}$, but shows no kinematic disturbance. The offset spectrum only extends to $\sim R_{25}$.

- ESO 473-G025 (Fig. 6), the brightest object in our sample $(M=-20.3)$, is an Sb-type galaxy at $\mathrm{cz}=7110 \mathrm{~km} / \mathrm{s}$. Acquisition images show what is at most a very subtle warp. The major axis spectrum extends a little beyond $R_{25}$. The kink visible in the rotation curve in Fig. 6 corresponds to a region where two components of the $\mathrm{H} \alpha$ line are visible at the same position in projection along the slit. However, there are no signs of kinematic anomalies towards the edge of the disk. The offset rotation curve is much less extended. A single emission region around $1.4 \mathrm{R}_{25}$ coincides with what appears to be a small satellite galaxy on the acquisition image.

- ESO 340-G026 (not shown due to space constraints) is an Sc-type galaxy at $\mathrm{cz}=5483 \mathrm{~km} / \mathrm{s}$ with $M=-19.5$. There are low-surface-brightness tails at the edge of the disk, but they show no significant warp. We exposed in two different offset positions, but obtained sufficient data for an analysis in only one case. The major axis spectrum, in any case, extends to $1.3 \mathrm{R}_{25}$ and thus far enough to sample the kinematics of the outlying features. The rotation curve, again, shows no signs of kinematic anomalies.

\section{Conclusions}

In an effort to measure the kinematics of gas in the warps in the outer disks of galaxies with high spatial resolution, we have targetted seven edge-on galaxies, most of them with known optical warps, using multi-hour long slit spectroscopy.

- In at least three of seven cases, we have observed more extended $\mathrm{H} \alpha$ emission if the slit was aligned along the warp rather than along the major axis. This shows that we have been successful at detecting gas in the warps, and that we have thus attained our observational goal. Counting the single emission region detected in UGC 12423, this ratio is four out of seven.

- In three of these four cases, there are no signs of kinematic anomalies in the rotation curve associated with the onset of the warp. 
- In one of these four cases, there is a sharp break in the rotational velocity roughly at the onset of the warp. In this case, the optical appearance of the "warp" is highly suggestive of a tidal feature. However, the break occurs in the major axis rotation curve.

- In the remaining objects, the outer-disk features are sampled by the major-axis spectrum, but there is no evidence for kinematic anomalies in these regions.

Based on a small data set (four kinematic detections of extraplanar outer disk gas likely to be associated with a warp), we find evidence for kinematic anomalies only in the one case whose optical appearance strongly suggests a tidal feature. In other cases, the kinematics of the presumed warp gas are consistent with the extrapolation of the inner disk rotation curve. This sample is therefore not in support of the hypothesis that the outer, warped disks are in direct contact and exchanging angular momentum with material being newly accreted; however, the possibility cannot be ruled out yet that the real point of contact between the galaxy disk and accreted material lies at larger radii, and that the gas that we see at the onset of the optical warp has already settled into disklike kinematics. We propose to explore three avenues for further investigation: (i) Gas at larger radii, substantially beyond the optical warps, should be targetted; ideally, targets should thus be selected on the basis of HI maps where available. (ii) The sample should be increased in size from the present three clear detections. (iii) Additional indicators that might constrain the origin of the warp gas, in particular, metallicity, should be included in the analysis.

Based on observations made with ESO Telescopes at the Paranal Observatories under programme ID <079.B-0426>.

\section{References}

Binney, J. 1992, ARA\&A ),30, 51

Binney, J., Jiang, I.-G., \& Dutta, S. 1998, MNRAS 297, 1237

Bland-Hawthorn, J., Freeman, K. C., \& Quinn, P. J. 1997, ApJ ), 490, 143

Bosma, A. 1981, AJ, 86, 1791

Briggs, F. H. 1990, ApJ, 352, 15

Christlein, D. \& Zaritsky, D. 2008, ApJ ), 680, 1053

Cox, A. L., Sparke, L. S., van Moorsel, G., \& Shaw, M. 1996, AJ, 111, 1505

Dekel, A. \& Shlosman, I. 1983, IAUS, 100, 187

Dubinski, J. \& Kuijken, K. 1995, ApJ, 442, 492

Hunter, C. \& Toomre, A. 1969, ApJ, 155, 747

Jiang, I.-G. \& Binney, J. 1999, MNRAS ), 303, 7

Kuijken, K. 1991, ApJ, 376, 467

Lynden-Bell, D. 1965, MNRAS, 129, 299

Nelson, R. W. \& Tremaine, S. 1999, MNRAS, 306, 1

New, K. C. B., Tohline, J. e., Frank, J., \& Vaeth, H. M. 1998, ApJ, 503, 632

Ostriker, E. C. \& Binney, J. 1989, MNRAS, 237, 785

Revaz, Y.\& Pfenniger, D. 2004, A\&A, 425, 67

Sancisi, R. 1976, A\&\&A, 53, 159

Sanchez-Saavedra, M. L., Battaner, E., \& Florido, E. 1990, MNRAS, 246, 458

Sanchez-Saavedra, M. L., Battaner, E., Guijarro, A., López-Correidora, M., \& Castro-Rodríguez, N 2003, A\&BA, 399, 457

Shen, J. \& Sellwood, J. A. 2006, MNRAS ), 370, 2

Sparke, L. S. \& Casertano, S. 1988, MNRAS, 234, 873

Toomre, A. 1983, IAUS, 100, 177 